\title{
Effect of Watermelon (citrullus lanatus) on pulse rate and blood pressure in healthy individuals
}

\author{
OluwoleF.S ${ }^{1,2}$, Adebayo- Gege G.I ${ }^{2}$, Abaya. J. G ${ }^{2}$ Atsukwei D ${ }^{2}$ \\ ${ }^{I}$ Department of Physiology, College of medicine, University of Ibadan, Ibadan, Nigeria \\ ${ }^{2}$ Department of Physiology, Bingham University, Karu.
}

\begin{abstract}
Watermelon is really useful, healthy and its great properties are well known to the world since ancient times. It has been reported that watermelon has an ingredient that deliver Viagra-like effects to the body's blood vessels and may even increase libido. This work was done to determine the effect of watermelon on pulse rate and blood pressure in healthy individuals. A total number of 40 adult human beings (20 males, 20 females) were randomly selected for use in this experiment. Watermelonwas given to each subject daily for seven days of experimental period respectively. The pulse rate was taken before and after the meal using palpation and the blood pressure was measured by auscultatory method.

In the female subject, there is significant $(P<0.01)$ increase between the basal pulse rate and the post watermelon pulse rate $(75.7 \pm 1.38$ to $82.7 \pm 2.58)$ showing a significant increase in pulse rate after taking the watermelon. In male subject, there is also significant $(P<0.01)$ increase between the basal pulse rate and post watermelon pulse rate after the experiment( $69.9 \pm 0.79$ to $77.9 \pm 1.38)$ showing a significant increase $(P<0.01)$ in pulse rate after taking the watermelon.there was no significant changes in the basal systolic pressure and post- watermelon systolic pressure in both male and female subjects. The basal diastolic blood pressure in the male subject slightly reduced compared with post watermelon diastolic while tin female subject,there is slight increase.The results of this experiment suggest that increase in the pulse rate is due to the effect of watermelon on the blood flow which may be due to action of l-citrulline (active ingredient) on the blood vessel enhancing the activity of the heart. There is no significant change on the blood pressure.
\end{abstract}

Keywords: watermelon, pulse rate, adult human beings, blood flow, blood pressure.

\section{Background}

Watermelon (Citrullus lanatus., Thunb family Cucurbitaceae) is a vine-like scrambler and trailer flowering plant originally from southern Africa. Its fruit, which is also called watermelon, is a special kind referred to by botanists as a pepo, a berry which has a thick rindexocarp and fleshy centermesocarp and endocarp. It contains phosphorus, calcium, iron, pectin, folic acids, vitamin $\mathrm{B}_{1}, \mathrm{~B}_{2}$, fructose and glucose, and all of them have proven beneficial action for the whole organism and weight loss effort (Aliya,2011, Anon, 2010b). Beneficial ingredients in watermelon are known as phyto-nutrients, naturally occurring compounds that are bioactive, or able to react with the human body to trigger healthy reactions. The citrulline which exists in watermelon is a known stimulator of nitric oxide, is an ubiquitous amino acid in mammals, isclosely related to L-arginine (Bredt and Synder,1990). In thekidney, vascular endothelium, and other tissues, L-citrulline can be readily converted to L-arginine, providesrecycling pathway for the conversion of L-citrulline to nitricoxide (NO) via L-arginine (Solomonson et al., 2003). From previous studies, it was found that L-arginine can reduce heartdisease and pulmonary disease in rats and reverse the progressionof atherosclerosis in rabbits (Sheridan $e t$ al., 1998; Hayashi et al. 2005). Watermelon is a diuretic and was a homeopathic treatment for kidney patients before dialysis became widespread.Also,it was found that oral L-arginine supplementation decrease brachial Blood pressure via improved endothelial NO productionin adults with prehypertension and hypertension(Palloshi et al.,2004, Siani et al.,2000, Martina et al.,2008) However, in this study the effect of water melon on the pulse rate and blood pressure in healthy individual of Bingham University,Nasarawa state

\section{Materials And Methods}

A total of 40 adult human beings ( 20 males, 20 females) subjects were randomly selected for use in this experiment. BP measurements obtained using an Omron automated sphygmomanometer after participants wereseated for at least $10 \mathrm{~min}$ before the experiment. Subjects had noapparent cardiovascular or metabolic diseases assessed by medicalhistory. Exclusion criteria included regular consumption of L-citrulline/L-arginine rich foods or supplements, smoking, $\mathrm{BP} \geq 140 / 90 \mathrm{~mm} \mathrm{Hg}$, and chronic diseases. The Bingham University Human Subjects committee approved the experimental procedures,and written informed consent was obtained from all subjects.Subjects were asked to maintain their diet and exercise habitsduring the study period. Dietary habits were assessed by afood frequency questionnaire, whereas physical activity wasrecorded and reported. A day before the subject were enlightened more on the experiments after the initial screening process.Cardiovascular 
tests were performed in the morning in a quiettemperature-controlled room $\left(22-24{ }^{\circ} \mathrm{C}\right)$ after an overnightfast and avoiding caffeinated drinks, alcohol, and intense exercise for at least $24 \mathrm{~h}$ before the experiment

Anthropometrics: Height was measured using a stadiometer to the nearest $0.5 \mathrm{~cm}$, and body weight was measured using aSeca scale (Sunbeam Products, Boca Raton, FL) to the nearest0.1 kg. Body mass index was calculated as $\mathrm{kg} / \mathrm{m} 2$.After basal measurements, subjects were randomly assigned to receive watermelon $1 \mathrm{~g}$ once daily for seven(7) days.

Pulse rate: the pulse rate was measured by palpation method. The subject were made to sit comfortably and the pulse rate was determined by palpating the radial artery just above the wrist and the pulse rate was counted 1560 secondsThe pulse is usually felt where an artery is compressed against a bone or near the skin.

\section{Statistical Analysis;}

The values are recorded as Mean \pm SEM at $\mathrm{p}<0.01$ significant difference using student $t$-test.

\section{Result}

Basal measurements and post-treatment measurement of pulse rate,systolic and diastolic blood pressure are shown in table I and II. There were significant changes in the pulse rate, there was increase in the pulse rate from75.7 \pm 1.38 beats $/ \mathrm{min}$ to $82.7 \pm 2.58, P<0.01$ in the female subject while in male subjects, it increases from $69.9 \pm 0.79$ to $77.9 \pm 1.38, \mathrm{p}<0.01$ after taking watermelon for 7 days. The systolic blood pressure in the female increases from $113 \pm 2.52 \mathrm{mmHg}$ to $120.0 \pm 1.62 \mathrm{mmHg}$, and the diastolic pressure $76.5 \pm 1.09 \mathrm{mmHg}$ to $85.5 \pm 1.14 \mathrm{mmHg}$.in the male subject, there was no significant difference in the systolic blood pressure $(\mathrm{P}>0.01)$ in the basal systolic blood pressure and post treatment blood pressure measurement. There was slight increase in the diastolic blood pressure in the male subjectsas shown in Table III

\section{Discussion}

This study examined the 7days of watermelon administration (containing natural L-citrulline and Larginine)in healthy individuals increases the pulse rate in both male and female subjects.this increase in pulse rate might be due to the effect of watermelon on the blood flow. Watermelon increases blood flow and reduces blood pressure by the action of l-citrulline on the blood vessels. The l-citrulline found in watermelon is a known stimulator of nitric oxide. Nitric oxide is thought to relax (vasodilator) and expand blood vessels to improve blood flow and reduce blood pressure, much like the erectile dysfunction drug Viagra, and may even increase libido. NO is a biologically active substance produced through theconversion of L-arginine to L-citrulline via a Ca2+-dependent cNOS or a Ca2+-independent iNOS (Nishida et al., 1998). This enhances the activity of the heart by increasing heart rate and beat which leads to the increased pulse rate.

Blood flow or blood pressure is directly affected by pulse rate or heart rate. Pulse is generated by heart activity, contraction and relaxation. In the contraction period, blood is pumped out of heart to lungs or systemic circulation, while in relaxation period, blood enter the heart. The time when the heart contract is called systole, which is the highest blood pressure of an individual can reach. The time when the heart relaxes is called diastole, a period before the heart starts to contract again. This creates pulsatory blood flow. The pulse per se is the time when the heart contracts, pushing blood to flow into circulation. There was significant $(\mathrm{P}<0.01)$ increase between pulse rate before and after breakfast i.e. between pre-watermelon pulse rate and post watermelon morning pulse rate in both female and male subjects from $78.0 \pm 1.63$ to $85.8 \pm 1.39$ and $69.5 \pm$ 0.56 to $78.9 \pm 1.47$ respectively. Blood flow is also increased after meals. This is as a result of increased metabolic activity leading to increased heart activity, heart rate and heart beat.Previous studies have observed reduction in brachial BP after oral L-arginine supplementation in adults with high BP and concurrent chronic diseases(Martina et al.,2008).

It was also found that 6 weeks of watermelon supplementationimproved aortic hemodynamic in middle-agedadults with prehypertension. In this study, watermelon does not show significant effect in the subjects. In the female the systolic blood pressure and diastolic blood pressure rise slightly after the $7^{\text {th }}$ day of postwatermelon and with no significant changes in the post watermelon in the morning and at $6 \mathrm{pm}$ but rather stabilizes the blood pressure.also in the males, there was no significant changes in both the systolic and diastolic blood pressure.

This has a little agreement with studies of Figueroa and others, 2010 that. watermelon did not alter $\mathrm{HR}$ (heart rate), $\operatorname{Tr}(\operatorname{Transit}$ time), and aortic PWV, and a decrease in ALx(augmentation pressure) whh was said to be more likely influenced bythe decrease in P2(first systolic peak). The result of this study therefore shows that in the healthy individuals, watermelon does not alter the blood pressure but rather stabilizes it and the pulse rate by relaxing the vessel and improving the blood flow which enhances the efficiency of heart pumping activity.Watermelon activity is therefore suggested to be via L-citrulline-NO cycle.

\section{Conclusion:}


It is therefore concluded that watermelon can stabilize the blood pressure and enhances the efficiency of the heart in healthy individuals. Its activity is suggested to be via L-citrulline and nitrogen cycle.

Table I: Showing the mean pulse rate values of both subject.

\begin{tabular}{|c|c|c|}
\hline \multirow[t]{2}{*}{ Grouping } & \multicolumn{2}{|l|}{ Mean Pulse rate $\quad$ Mean pulse rate } \\
\hline & $\begin{array}{r}(\text { Mean } \pm \text { SEM }) \\
\text { Female }\end{array}$ & $\begin{array}{r}(\text { Mean } \pm \text { S.E.M.) } \\
\text { Male }\end{array}$ \\
\hline iBasal pulse rate & & $75.7 \pm 1.38$ \\
\hline ii Pre watermelon(Before breakfast) & $78.0 \pm 1.63 * *$ & $69.5 \pm 0.56$ \\
\hline iii Post water melon (morning) & $85.8 \pm 1.39 * *$ & $78.9 \pm 1.47 * *$ \\
\hline iv Post water melon (At 6.00 pm) & $80.0 \pm 1.36 * *$ & $74.8 \pm 0.82 * *$ \\
\hline $\mathrm{v}$ Post water melon (After 7 days) & $82.7 \pm 2.58 * *$ & $77.9 \pm 1.38 * *$ \\
\hline
\end{tabular}

** $(p<0.01)$ shows a significant difference when basal pulse rate is compared with pulse rate post watermelon.

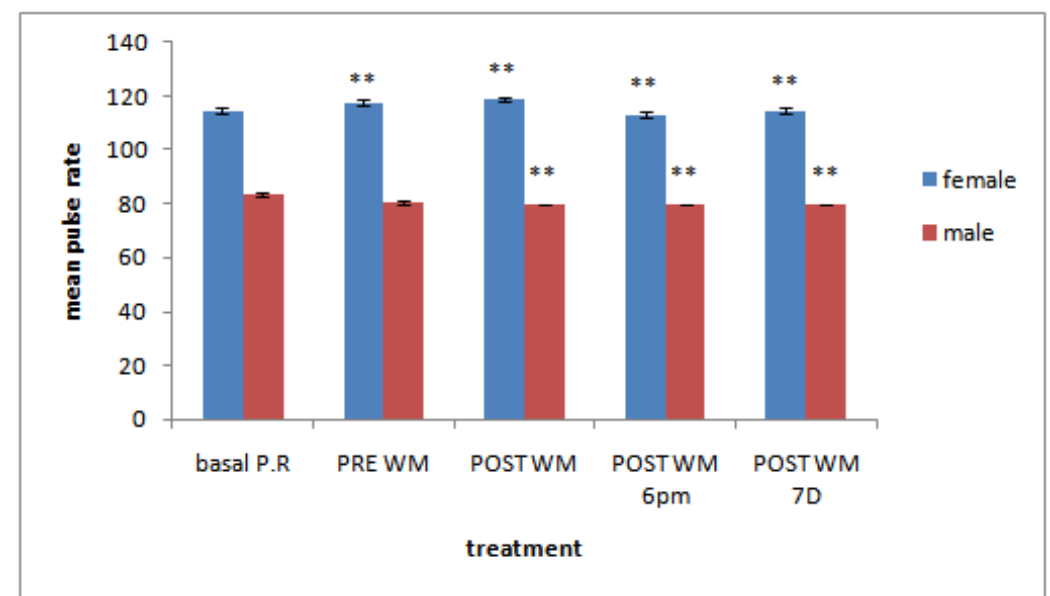

Fig I. Graph showing the mean pulse rate values of both subjects treated with Water melon (WM).P.R=Pulse rate, $7 \mathrm{D}=7$ Days., $\mathrm{p}<0.01$

Table II: Showing the Systolic and Diastolic Blood pressure values of female subjects.

\begin{tabular}{|c|c|c|c|c|}
\hline Treatment & \multicolumn{3}{|c|}{$\begin{array}{l}\text { Systolic B.P Diastolic B.P } \\
\text { (Mean } \pm \text { SEM) }\end{array}$} & $(\mathrm{Me}$ \\
\hline iBasal Blood pressure & \multicolumn{3}{|c|}{$113 \pm 2.52$} & $76.5 \pm 1.09$ \\
\hline ii Pre watermelon(Before breakfast) & \multirow{3}{*}{\multicolumn{2}{|c|}{$\begin{array}{r}119.5 \pm 2.23 \\
\quad 122.0 \pm 1.72\end{array}$}} & $82.0 \pm 1.38$ & \\
\hline iii Post water melon (morning) & & & & $83.5 \pm 1.09$ \\
\hline iv Post water melon (At $6.00 \mathrm{pm}$ ) & & & $77.5 \pm 0.99$ & \\
\hline v Post water melon (After 7 days) & \multicolumn{2}{|c|}{$120.0 \pm 1.62$} & & $85.5 \pm 1.14$ \\
\hline
\end{tabular}

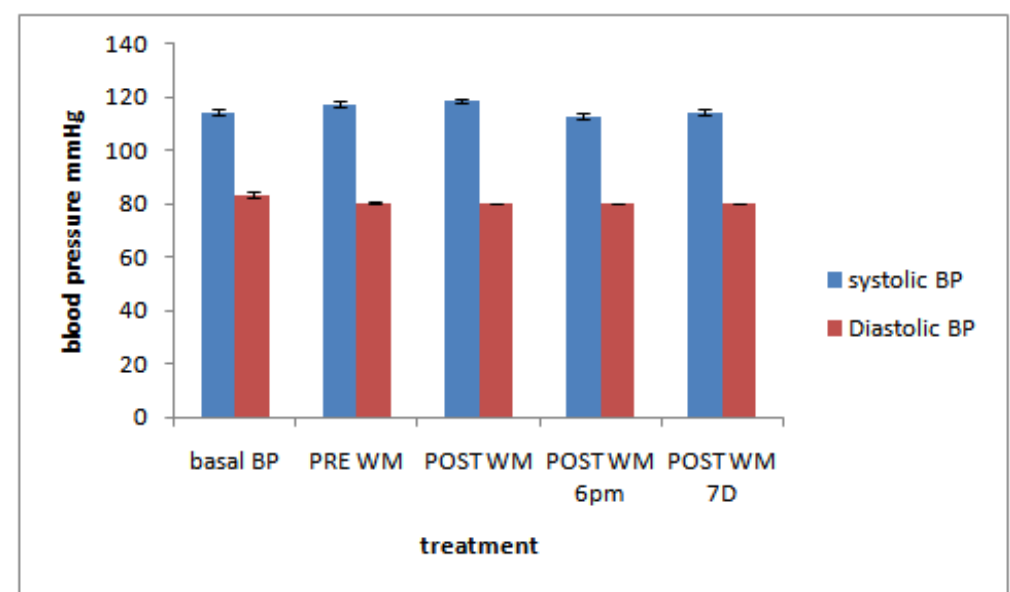

Fig II: Graph showing the Systolic and Diastolic Blood pressure values of female subjects treated with Water melon (WM), B.P=Blood pressure, 7D=7Days.

Table III: Showing the Systolic and Diastolic Blood pressure values of male subjects. 
Effect of Watermelon (citrullus lanatus) on pulse rate and blood pressure in healthy individuals

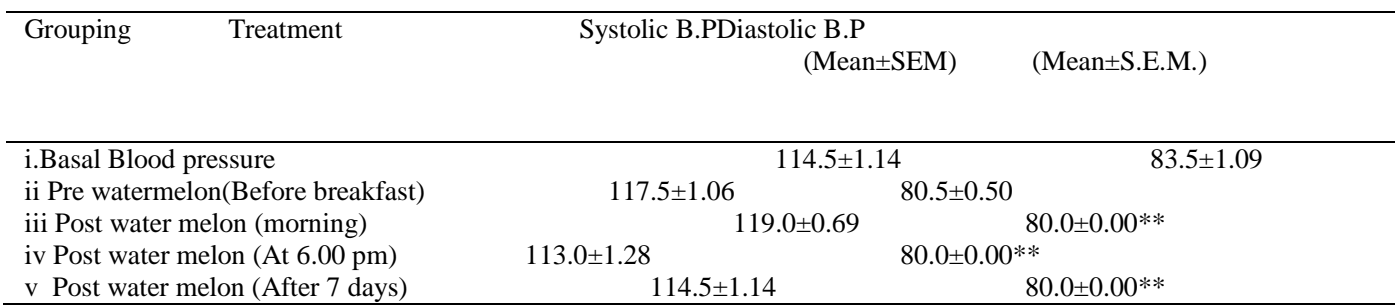

** $(\mathrm{p}<0.01)$ shows a significant difference when basal diastolic blood pressure is compared with post watermelon diastolic blood pressure.

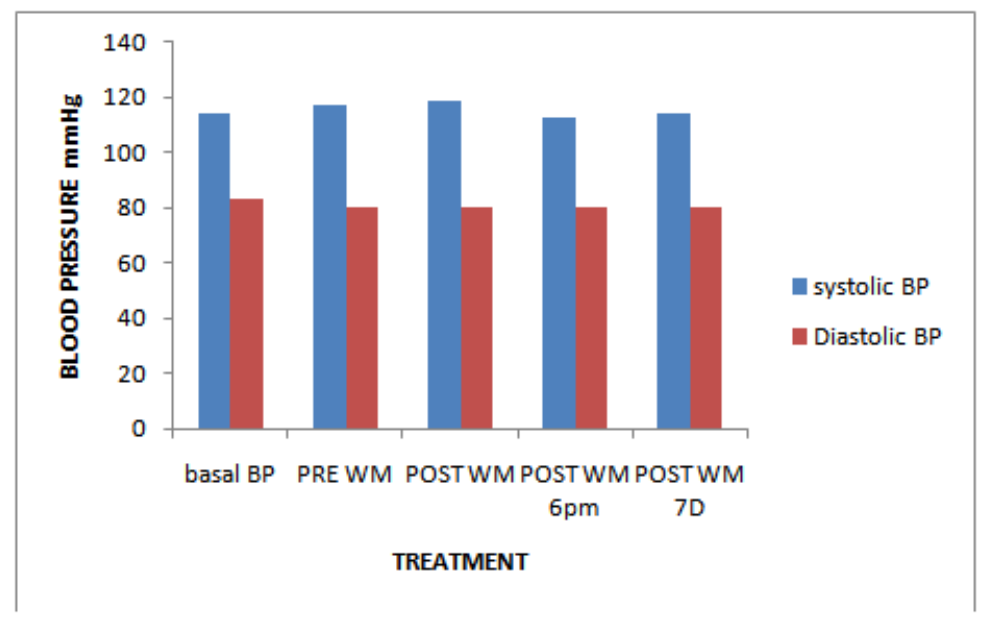

Fig III: Graph showing the Systolic and Diastolic Blood pressure values of male subjects treated with Water melon (WM) B.P=Blood pressure, 7D=7Days.

\section{References}

[1] Anon. National Watermelon Promotion Board.visited May 2010b. www.water.org

[2] Bredt, D.S., and Snyder, S.H.. Isolation of nitric oxidesynthase, a calmodulin-requiring enzyme. Proc. Natl. Acad. Sci.U.S.A. 1990, 87(2): 682-685. doi:10.1073/pnas.87.2.682. PMID: 1689048.

[3] Solomonson, L.P., Flam, B.R., Pendleton, L.C., Goodwin, B.L., and Eichler, D.C. 2003.The caveolar nitric oxide synthase/arginine regeneration system for NO production in endothelial cells. J. Exp.Biol. 206(12): 2083-2087.

[4] Sheridan, B.C., McIntyre, R.C., Jr, Meldrum, D.R., and Fullerton, D.A. 1998. L-arginine prevents lung neutrophil accumulation andpreserves pulmonary endothelial function after endotoxin. Am. J.Physiol. 274(3 Pt. 1): L337-L342. PMID:9530168

[5] Hayashi, T., Juliet, P.A.R., Matsui-Hirai, H., Miyazaki, A., Fukatsu,Izhar, U., Schwalb, H., Borman, J.B., and Merin, G..Cardioprotective effect of L-arginine in myocardial ischemia andreperfusion in an isolated "working rat heart model. J. Cardiovasc.Surg. (Torino), 1998, 39(3): 321-329. PMID:9678555.

[6] Palloshi A, Fragasso G, Piatti P, Monti LD, Setola E, Valsecchi G, Galluccio E,ChierchiaSL, Margonato A. Effect of oral Larginine on blood pressure andsymptoms and endothelial function in patients with systemic hypertension,positive exercise tests, and normal coronary arteries. Am J Cardiol2004; 93:933-935.

[7] Siani A, Pagano E, Iacone R, Iacoviello L, Scopacasa F, Strazzullo P. Blood pressure and metabolic changes during dietary Larginine supplementation in humans.Am J Hypertens2000; 13:547-551

[8] Martina V, Masha A, Gigliardi VR, Brocato L, Manzato E, Berchio A, MassarentiP,Settanni F, Della Casa L, Bergamini S, Iannone A. Long-term $\mathrm{N}$-acetylcysteinenand L-arginine administration reduces endothelial activation and systolic bloodpressure in hypertensive patients with type 2 diabetes. Diabetes Care 2008;3:940-944

[9] Nishida, K., Ohta, Y., and Ishiguro, I.. Changes in nitric oxideproduction with lesion development in the gastric mucosa of ratswith water immersion restraint stress. Res. Commun. Mol. Pathol.Pharmacol. 1998, 100(2): 201-212. PMID:9667074.

[10] Arturo Figueroa1, Marcos A. Sanchez-Gonzalez1, Penelope M. Perkins-Veazie2 and Bahram H. Arjmandi12010:Effects of Watermelon Supplementation on AorticBlood Pressure and Wave Reflection in IndividualsWith Prehypertension: A Pilot Study American Journal of Hypertension, advance online publication doi:10:142 